Unfallchirurg $2011 \cdot 114: 284$

DOI 10.1007/s00113-011-1959-0

(c) Springer-Verlag 2011

D.W. Sommerfeldt · H.-G. Dietz

Abteilung für Kinder- und Jugendtraumatologie,

Altonaer Kinderkrankenhaus gGmbH, Hamburg

\title{
Kinder- und Jugendtraumatologie - obere Extremität
}

erkannten Verletzung oder inadäquaten Therapie [Dtsch Arztebl Int 2009; 106(30):491-498]. nehmende Dynamik hin zu einer Superspezialität der Orthopädie/Unfallchirurgie bzw. der Kinderchirurgie. Vor allem durch die Zusammenführung der Fachdisziplinen Orthopädie und Unfallchirurgie ist klar geworden, dass die gesamte Breite der Diagnostik und Therapie, der konservativen und operativen Möglichkeiten am Skelett- und Bewegungsapparat im Kindesalter in der Zukunft wohl nur noch von einem Spezialisten angeboten und kompetent vertreten werden kann. Die Einrichtung von eigenständigen Organisationseinheiten der Kinderund Jugendtraumatologie in kinder- und unfallchirurgischen Kliniken ist noch am Anfang. Eine ständige Zunahme der Teilnehmer an Tagungen, Weiterbildungsveranstaltungen und Workshops zum Thema Kinder- und Jugendtraumatologie spricht in jedem Fall für einen gestiegenen Bedarf an Fort- und Weiterbildung auf diesem Sektor.

Für viele Unfallchirurgen, Kinderchirurgen und (Kinder-)Orthopäden ist die Kinder- und Jugendtraumatologie eine Bereicherung ihres klinischen Spektrums, die neue und andere Herausforderungen bereit hält. Skelettdiagnostik an Kindern ist indikatorisch nicht trivial, therapeutisch finden sich jedoch Verletzungsmuster, die relativ einheitlich sind und in der Regel ohne größere technische Herausforderungen bewältigt werden können. Für manche ist die Kinder- und Jugendtraumatologie allerdings auch ein Buch mit sieben Siegeln, mit Ossifikationszentren, Wachstumsstörungen und anderen Komplikationen bis hin zum Regress bei einer nicht

\section{Ziel ist die Sicherstellung der qualitativ hochwertigen, flächendeckenden und wohnortnahen Versorgung der häufigeren Verletzungen im Wachstumsalter}

Aus unserer Sicht kann die Lösung für solche Probleme nun nicht die Bildung von wenigen, überregionalen Zentren der Kinder- und Jugendtraumatologie sein, die dann alle Verletzungen des Kindes und des Jugendlichen behandeln, sondern die Sicherstellung der qualitativ hochwertigen, flächendeckenden und wohnortnahen Versorgung der häufigeren Verletzungen im Wachstumsalter. Es ist Aufgabe der Fachgesellschaften und Berufsverbände diese Versorgungsqualität durch ein attraktives Fort- und Weiterbildungsangebot herzustellen. Für wenige, genau zu definierende Verletzungen sollten allerdings in der Tat Anlaufpunkte in Form von Kindertraumazentren definiert werden, die ohne große bürokratische Hürden bei Problemen gezielt und v. a. zeitnah in Anspruch genommen werden können.

Die Sektion Kindertraumatologie der DGU als interdisziplinäre Arbeitsgruppe von Kinderchirurgen, Unfallchirurgen und Orthopäden versteht sich als ein Teil des oben geforderten Fort- und Weiterbildungsangebots. Durch regelmäßige Jahrestagungen zu relevanten Themen, durch die Organisation von Workshops, durch die Erstellung von Leitlinien und Lehrbüchern sowie die Publikation wissenschaftlicher Artikel soll es gelingen, sowohl die wohnortnahe Standardversorgung als auch die Spezialbehandlung im Zentrum qualitativ zu verbessern.

In diesem Sinn sind auch diese beiden Ausgaben des Unfallchirurgen zu verstehen. Wir haben hier, jeweils zur oberen und zur unteren Extremität relevante und z. T. auch strittige Themen bzw. Verletzungen gewählt, die von Unfallchirurgen, Orthopäden und Kinderchirurgen gleichermaßen behandelt werden. Keiner der Artikel versteht sich jedoch als Dogma, sondern vielmehr als Beitrag zum wissenschaftlichen Diskurs. Insofern freuen wir uns auch auf kritische Leserbriefe zu den einzelnen Beiträgen, denn wo diskutiert wird, ist auch Leben und die Kinderund Jugendtraumatologie ist mit Sicherheit eines: lebendig.

Für die Sektion Kindertraumatologie der DGU e.V.

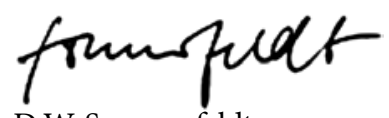

D.W. Sommerfeldt

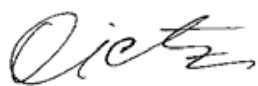

H.-G. Dietz

\section{Korrespondenzadresse}

PD Dr. D.W. Sommerfeldt

Abteilung für Kinder- und Jugendtraumatologie,

Altonaer Kinderkrankenhaus gGmbH Bleickenallee 38, 22763 Hamburg dirk.sommerfeldt@kinderkrankenhaus.net 